\title{
"You Can't Feel Healthier than Your Caregiver" - The Ripple Effect of Trust and Empathy for Patients and Health Care Staff, Cultivated through Cultural Activities
}

\author{
Christina Grape Viding ${ }^{1^{*}}$, Walter Osika ${ }^{2,3}$ and Eva Bojner Horwitz ${ }^{1-3^{*}}$ \\ ${ }^{1}$ Department of Public Health and Caring Sciences, Uppsala University, Uppsala \\ ${ }^{2}$ Center for Social Sustainability, Inst. of Neurobiology, Care Sciences and Society, Karolinska Institutet, Stockholm \\ ${ }^{3}$ Department of Clinical Neuroscience, Karolinska Institute, Stockholm, Sweden
}

*Corresponding author: Horwitz EB, Department of Clinical Neuroscience, Karolinska Institutet, Norra Stationsgatan 69, v 7 , 113 64 Stockholm, Sweden Tel: +46705863694; E-mail: Eva.bojner.horwitz@ki.se

Received date: July 12, 2017; Accepted date: August 22, 2017; Published date: September 02, 2017

Copyright: @ 2017 Viding CG, et al. This is an open-access article distributed under the terms of the Creative Commons Attribution License, which permits unrestricted use, distribution, and reproduction in any medium, provided the original author and source are credited

\begin{abstract}
Introduction: Stress-related problems, including burnout, cause personal suffering, disability and result in costly sick leave. The use of different cultural activities within a health care system may help to prevent burnout, but very few studies have focused on what happens after such activities.

Methods: Interventions with different cultural activities were conducted for burn out patients in four primary health care settings during three months. Focus group interviews were conducted with patients, cultural producers and health care nurses. The aim was to provide a description of the participant's experiences after the activities.

Results: The cultural activities were found to affect both participants' emotions and behaviour and created a sense of belonging and equality among them. Positive 'spill-over' effects were also seen on nurses of health care management.

Conclusion: We find that the cultural activities help to create a trustful and empathic health care environment where the wellbeing of staff and that of patients have an impact on each other. We suggest the incorporation of arts into health care as a possible contributor to the development of a sustainable health care system.
\end{abstract}

Keywords: Burnout patients; Cultural activities; Cultural palette; Empathy; Focus groups; Nurses; Phenomenological hermeneutic; Primary health care; Trust

\section{Introduction}

A significant portion of health care personnel report high levels of work-related stress, fatigue and mental disorders including burnout, which is the leading cause of disability and associated sick leave in Sweden [1-3]. For example, nurses have high rates of stress and fatigue both during their time studying and early on in their careers $[4,5]$. Work-related stress not only causes individual suffering, but is also responsible for costly sick leave, especially in health care [2]. Workrelated stress is thought to be one of the main causes of burnout [6-10]. If health care personnel are experiencing high levels of stress and burnout, this is likely to negatively impact the quality of care that they can provide and subsequently affect levels of patient satisfaction [11-13].

Cultural activities have the potential to provide both physiological and emotional benefits to individuals who suffer from stress, and are therefore worthy of consideration in strategies that aim to prevent and treat stress-related problems. In a recent study, The Cultural Palette, we show that cultural activities have positive effects in treating burnout symptoms in female patients [14], furthermore a large-scale longitude cohort study with participants from the Swedish working population show that workplaces with access to cultural activities during work hours seem to have protective effects against burnout [15]. Several studies show that cultural activities can stimulate emotions and behaviours that increase quality of life [16-19]. Cultural activities can enrich and enhance our memory and stimulate new neural pathways, they enable us to accelerate learning and to better comprehend our feelings, giving them meaning and context [20,21]. Cultural activities have also been shown to improve physical health, social function and vitality among health care staff [22]. Other initiatives, such as the Arts in Medicine (AIM) program, have been shown to reduce stress and increase revitalisation among nurses in their working environment [23].

Despite this growing body of research, the number of cultural and arts programs has recently increased in clinical health settings [24]. The arguments that the arts are an important contributor to human well-being are convincing, but the lack of robust empirical evidence has made it difficult to evaluate non-pharmacological, art-focused interventions in health care [25]. Quantitative evaluations of health outcomes, calculations of cost savings, and qualitative evaluations of how the health care system responds to cultural activity interventions are much needed. We will therefore in this study present the qualitative data on the previous reported quantitative analyses [14] after The Cultural Palette in primary health care settings. 


\section{Aim}

The aim of this study is to provide a description of the patients, the cultural producers and the primary health care nurses experiences, after The Cultural Palette.

\section{Methods}

The qualitative data in this study was collected following a quantitative randomised control intervention study, called the Cultural Palette involving six different cultural activities (Table 1) in four health care centres in Stockholm, Sweden [14]. A KEDS scale (Karolinska Exhaustion Disorder Scale) was used to evaluate burn out symptoms, and a cut off over two on the KEDS scale was used as inclusion criteria [14]. The Palette consisted of a wide range of activities including: interactive theatre, movie showings, vocal improvisation and drawing, explorative dance, mindfulness and contemplation, and a musical show. All the activities representing different modalities and senses [14]. The four different health care centres presented the same cultural activities with the same cultural producers. For an explanation of each cultural activity (see Table 1).

\begin{tabular}{|c|c|c|}
\hline S. No. & Activities & Cultural Palette Study \\
\hline 1 & Interactive theatre & $\begin{array}{l}\text { An experienced actor introduced poetical lyrics and poems and then initiated and participated in discussions with } \\
\text { the participants regarding thoughts, emotions, and experiences evoked by the texts. }\end{array}$ \\
\hline 2 & Movie showings & $\begin{array}{l}\text { After showing a movie, a film expert initiated discussions among the participants about experiences and thoughts } \\
\text { evoked by the movie. }\end{array}$ \\
\hline 3 & Vocal improvisation and drawing & $\begin{array}{l}\text { After participating in a vocal improvisation session with an experienced performance artist and pianist, the } \\
\text { participants painted a picture representing emotions, thoughts and pictures evoked during the improvisation. }\end{array}$ \\
\hline 4 & Explorative dance & $\begin{array}{l}\text { The participants improvised dance movements under the guidance of a dance movement pedagogue and music } \\
\text { teacher. The dance movements were staged according to the layout of the room and with focus on bodily } \\
\text { awareness. Afterwards the group discussed their experiences during the dance session. }\end{array}$ \\
\hline 5 & Mindfulness and contemplation & $\begin{array}{l}\text { The participants contemplated and practiced mindfulness together with an experienced mindfulness instructor. } \\
\text { Attention was on breathing and body awareness. Thoughts, feelings, images and sensations were in focus and } \\
\text { experiences were reflected in the group after the contemplation. }\end{array}$ \\
\hline 6 & Musical show & $\begin{array}{l}\text { After a musical show including music, song and dance focusing on bodily awareness, the participants discussed } \\
\text { their thoughts regarding the body with the actor. }\end{array}$ \\
\hline
\end{tabular}

Table 1: The table shows the different cultural activities involved in the cultural palette study. Each activity was presented twice, which means that during the 12 weeks of intervention, the patients received 12 activities in total.

The cultural activities were offered as a package once a week, lasting 90 min per session, for three months. They took place in the conference room at each health care centre. The results showed decreased levels of exhaustion, increased ability to express emotions, and increased levels of self-rated health in the patient group [14].

After the four separate Palette interventions at the four respective health care centres, the following five focus groups and four individual interviews were conducted:

- Four focus groups with burned out patients from each of the four health care centres

- One focus group with all the cultural producers from the Cultural Palette, and

- Four individual interviews with nurses in managerial positions representing each health care centre.

The characteristics of the focus groups members were diverse and represented different personalities with both introvert and extrovert personalities. Therefore, the interviewer put the questions in a certain system so everybody could share experiences from the Cultural palette.

All the participants were asked the following question: How did you experience the Cultural Palette? Follow-up questions regarding the participant's experiences were made throughout the interviews. When no additional data could be found to develop new properties of categories, the researcher was empirically confident that the categories were saturated. The rich experience in focus group work helped the researcher to be able to determine the depth of the data and therefore to determine the criterion of saturation.

The texts from the five focus groups and the four individual interviews were transcribed verbatim and analysed thematically in accordance with a phenomenological hermeneutic method [26,27].

\section{Participation Rate}

There were a total of 32 participants in this study, consisting of: 22 patients $(5,7,4,6$, respectively in each patient focus group), 6 cultural producers (in the cultural producer focus group) and four nurse managers.

\section{Data Analysis}

The analytical lens that was used to conduct and interpret both focus group and individual interviews is based on a methodology inspired by the French philosopher Paul Ricoeur - we refer to this as a phenomenological hermeneutic method [24,26,27]. The method regards the written text as a source of meaning about the phenomena in question. This method treats the text as a mode of recording lived experience that can subsequently be interpreted through a number of steps or 'levels' [27].

The following levels facilitate the analysis 
Citation: Viding CG, Osika W, Horwitz EB (2017) "You Can't Feel Healthier than Your Caregiver" - The Ripple Effect of Trust and Empathy for Patients and Health Care Staff, Cultivated through Cultural Activities. J Nurs Care 6: 422. doi:10.4172/2167-1168.1000422

Page 3 of 8

1. 'Naïve Reading': the transcribed text from the interviews is read several times and are summarised with a concise description (Table 2).

2. 'Structural Analysis':

- The text is broken into 'meaning units', which are distinct phrases or assertions that are identified as relevant with respect to the research question.

- Meaning units are condensed, which requires their combination and concentration.

- From the process of condensing, sub-themes are derived.

- Themes are created to reflect how the groups of sub-themes are conceptually distinct from one another.

- The complete interpretation informs the analysis that follows.

3. The "Complete Interpretation": the result of all the processes at the above levels. It comprises the naive reading, the structural analysis along with notes on the researcher's preunderstanding, all of which are delimited with regards to their relevance to the research questions [24,26,27].

\section{Validity and Trustworthiness of the Data}

The primary analysis of the text was carried out by the first author and then peer-reviewed by the third author. All the authors have expertise in the Cultural Palette model and in culture/arts in health and have different professional background. This knowledge of the relevant theory, together with the three levels of analyses provided by the phenomenological hermeneutic model, helps to enhance the validity of the study and gives the Naïve Reading its relevant value. During the peer-review by the third author, a triangulation between the different evaluators was made possible and different perspectives of the participants experiences was made visible.

The interpretative nature of the qualitative analysis cannot draw on the notion of validity in the same way as in quantitative analyses because it elucidates the subjective meanings of the participant's experience of the cultural activities [26].

In this qualitative study, the dual perspective of the researcher's background help strengthens the validity. The focus groups together with the individual interview also helps to derive reliability from its use of multiple perspectives on the same phenomena; the Cultural palette.

To be able to control possible bias during and in the data collection and analysis, the study leader have randomly selected two participants from each focus group and one from the four individual interviews to read through the text and comment on the analyses in order to possibly contrast interpretation among researchers and participants.

To ensure reliability and validity of the study, the text has been checked, confirmed and processed regarding its certainty in relation to the analyses during the research process. The three authors have also read the final analyses without mutual influence and agreed on consensus.

\section{Naive Reading (Table 2)}

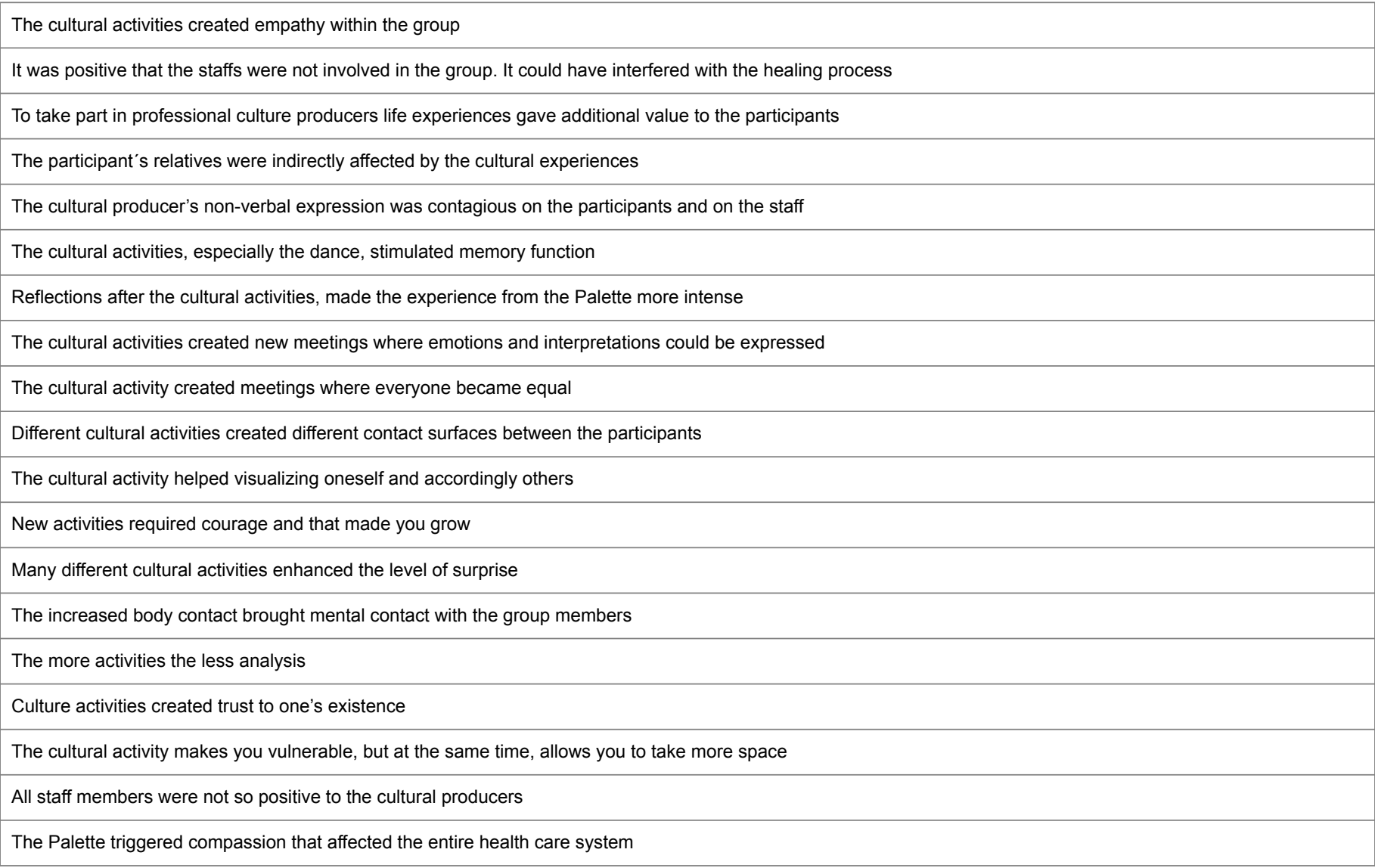


Citation: Viding CG, Osika W, Horwitz EB (2017) "You Can't Feel Healthier than Your Caregiver" - The Ripple Effect of Trust and Empathy for Patients and Health Care Staff, Cultivated through Cultural Activities. J Nurs Care 6: 422. doi:10.4172/2167-1168.1000422

Page 4 of 8

\begin{tabular}{|l|}
\hline Healing cannot occur in a sick environment \\
\hline Emotional expressions create the prerequisite for healing \\
\hline It was important that the Palette didn't disturb the working environment at the health care clinic \\
\hline The health care management became aware of how cultural activities can be used for future staff training \\
\hline Difficult for health care centre managers to find funding for cultural activities \\
\hline Important that caregivers are reinforced so they have the strength to care for patients \\
\hline
\end{tabular}

Table 2: Shows the summary of the Naïve reading, which is transcribed text from all the interviews summarised with a concise description.

\section{Results}

The results are presented per three themes:

- Cultural Palette as having an impact at the level of the body (physical, emotional and cognitive impact)

- Cultural Palette as having an impact at the level of the group

- Cultural Palette as having an impact at the level of the health care system

Supporting quotes, taken from the empirical data, are presented below. They are coded with the numbers 1-5 where each number represents each focus group. The different patient focus group represent number 1-4 and the cultural producer focus group number 5 . The quotes from the managers are coded with number 6 . The different participants speaking in each group are coded with letters a-f.

1. Cultural Palette as having an impact at the level of the body (physical, emotional and cognitive impact)

"I become vulnerable, while at the same time asserting myself (2a)

"You recall that you know more than you think you know, you can use so much more than you do" (3b)

"You have become more open to different interpretations; there are more interpretations than you knew before" (1c)

"Previously I just pushed things away, but now I look at things a little more objectively" (4d)

From these quotes, the engagement in the different cultural activities in the Palette seems to have an impact on the body, emotions and memory function. The first quote is particularly telling of the creative process that is instigated by the activities: the seeming paradox of becoming vulnerable and at the same time asserting oneself resonates with Csikszentmihalyi's definition of creativity as a 'flow [28]; a creative process where one is alert and stimulated but simultaneously focused and calm.

"I rediscover my body in a new way that I normally don't do" (1a)

"Cultural activity, a reference to earlier discoveries in life, awakens it" (5a)

"The producer's non-verbal expression was contagious on me and on rest of my staff" (6b)

"The dancer... has never been close to a person who had such rich body language, I absorbed her movements and it felt as though my memory came back, it was magic" (3d)

The interrelation between the cultural activity and the physical body is striking in the quotations and shows that openness is cultivated towards new interpretations, memory and trust. Moreover, the cultural activity stimulates emotions and helps the participant to identify emotions that they were previously unaware of. The physicality, the 'body', of the cultural activities appears to mirror and manifest themselves in the participants' bodies, also on the staff.

\section{Cultural Palette as having an impact at the level of the group}

"The group was small, courage was required. New things require more courage" (5b)

"How can you approach other people when the distance only increases? Here we could meet thanks to the cultural activity..." (1b)

"This has created a willingness to listen with empathy to other people, which you almost never do otherwise. Here it was okay to do so" (2b)

"The working climate was different, in a good way, after the Palette was here-more open" (6a)

The cultural activities created a unique dynamic for its participants and a feeling of togetherness enabling the 'whole person' to be brought to the group. Furthermore, this 'whole person' effect seemed to be maintained after the group interaction and it began in small ways to transform the participants' everyday lives. The willingness to listen empathically to each other increased over the period of the Palette and developed individuals' courage and built trust between the participants:

"We trust each other even though we don't know each other, don't need to know where we come from, trust" (2d)

"I let go of my values, I felt a huge sense of community. Wow, I thought...I felt warmth from the whole group, a craving from deep within, here I belong, I have tried things before but the message was shared by the whole group" (3c)

"We talk to each other about things that we/I don't talk about with those closest to me" (3a)

"You don't have to have the same symptoms, it doesn't matter, everyone has unique problems and that is the way in and we all gained courage" (1d)

A transformation of relations at the level of the group challenges the hierarchical culture that tends to characterise health care settings, democratising health care workers' interpretations of each other. Identification with the group that had formed around the Palette intervention reinforced trust, courage and curiosity among those who engaged in cultural activities, the cultural producers and members of healthcare management. This ripple effect of trust, curiosity and 
empathy can be seen at group level within the study, but there were clues that it may also have affected relatives and family members:

"My husband has been affected actually, with knock-on effects on the whole family" ( $3 b$ )

3. Cultural Palette as having an impact at the level of the health care system:

"The system has so many opportunities to change when there is compassion and in that sense cultural activity can make a difference" (6c)

\section{"You can't feel healthier than your caregiver" (2d)}

"The staff should participate in a group of their own; they have a different kind of burnout" (1c)

The Cultural Palette intervention provided an opportunity for all the participants to reflect on the health care system 'as a whole'. As we have seen, the cultural activities increased empathy not only among patients but also among health care management and may have made the organisations themselves more flexible and open to change. The interviews and focus groups revealed the importance of the health of the caregiver to the experience of the patient, and that the health of the caregiver worker was mirrored in them:

"People need to look up and smile in order for the healing to take place" (5d)

"It's positive that someone from outside the system influences the system, without disturbing the work" (6a)

"Cultural providers should come regularly; both for patients and for the staff... everybody needs a shot" (6d)

"Don't distinguish between patients and staff; everyone has the same value and the same right to feel good" (4c)

"Who are we to take care of our patients if we can't take care of ourselves" (6a)

In this theme, we see that the fundamental dynamic between patient and health care worker is at stake, the healing dynamic on which health care is built. While the first and second theme point to the benefits of the cultural activities at individual and a group level, the cultural activities seem also to be relevant to the transformation of the health care as a system. To achieve any of these effects, the conduct of an intervention such as the Palette requires time and space within work place: this also requires organisational change, something that extends beyond individual and group level.

\section{The Complete Interpretation}

In the final step, all texts are considered in relation to one another. It comprises the naive reading, the research question, the structural analysis and notes on the researchers' preunderstanding of the phenomenon. The result of these processes taken together is the complete interpretation. The complete interpretation developed from the present study is presented below:

\section{Cultural palette - Interaction of trust and empathy at bodily, group and system level}

The Palette promoted a new awareness for the participants about their health at bodily, group and system levels. In the individual (bodily) interaction with the cultural activities, feelings are awakened through actions and behaviours that patients would not otherwise engage in. Such engagement with the body facilitates the development of a healthy connection between one's own body and those of others in the group, allowing the patient to become more than their symptoms. A new kind of visibility of oneself emerges, whereby one can dare (courage) to show oneself while remaining vulnerable.

The Palette creates a link between the individual and the group. Connections are created not only among patients, but also between patients and staff in the health care centres. The feelings and behaviours that are awakened, aid to build stronger, empathic relations between people. The Cultural Palette thus entails transformation that ripples out not only beyond the individual patient to the level of the group, but also to a community level.

\section{Discussion}

The participants experienced that the Palette had a positive impact on their emotions, behaviours and that it gave them a sense of belonging. The Palette also demonstrated the value of uniting the exhausted, burnt-out patients, engendering in them a strong sense of group coherence. The participants started to reflect on how meaningful the unconditional meetings with other people were to them: "I let go of my values, I felt a huge sense of community. Wow, I thought, I felt warmth from the whole group, a craving from deep within, here I belong" and "You talk to each other about things that we/I don't talk about with those closest to me". The positive experiences of the Palette also spread to the health care management in a positive way. Even though the health care managers did not participate in the Palette themselves, they experienced a positive effect by virtue of having brought the beneficial experience into their health care environments.

In all, the results from this qualitative data analyses were very positive (as well as the results from the quantitative analyses, [14]) even thought it was challenging and time consuming to construct the different platforms of cultural intervention within the health care system. Thinking beyond this study, it is important to consider whether it is possible to replicate these positive results in other health care contexts. As defined by the Medical Research Council guidance (MRC guidelines), arts-in-health interventions are complex and often difficult to standardise into a broader context or organisation $[29,30]$, so establishing causal relationships between a local intervention with an outcome is difficult. One way of understanding and working with the complexity of a medical intervention (such as arts in health) is to continue collecting and analysing qualitative data together with the quantitative data such as that gathered in this Palette project [14]. Another way is to draw on theoretical frameworks in order to understand the relationships of the setting in question with a view to developing research within arts and health in future project planning $[31,32]$.

The following part of the discussion uses the literature to contextualise some of the most salient ideas that were raised in the study. Firstly, it is thought that when you have been exhausted or burnt-out for a long period of time, the cognitive systems and memory function are affected [6]. Emotion- and stress-processing networks seem to be disrupted [33]. The ability to control negative emotions is impaired, which can render you more vulnerable to depressive symptoms. As we have seen, cultural activities can enhance the contact we have with our emotions: "The mix of approaches helped me because I am usually an analytical person and getting away from such analysis helped release the blockage that I felt when I was affected". This quote 
communicates the notion of an ability to do things without analysis and instead letting the body be surprised. A similar idea is discussed in the theory development of 'the emotional brain' [34]. It seems that engagement in cultural activities allows for a bypassing of the analytical function to the emotional part of the brain thought being located in the amygdala. Another clue to this can be found in the following quote: "The blockage that I felt was released when I was affected, for example by the voice package." Increased capacity to regulate our emotions is thought to counteract depression [14]. This may have a positive impact not only on personal suffering but also on public health as a buffer against exhaustion if we use cultural activities regularly [15].

The working through of cognitive 'blockages' has been explored in recent research around writer's block, whereby it seems to be possible to harness the transformative power of contemplative movements facilitated through engagement with artistic practices [35]. It has been argued that multimodal stimulation can have a greater effect on the brain compared with stimuli from just one modality at a time, which means that brain activity increases when we stimulate using several culture modalities (such as auditory and visual), for example, with music and pictures together [36]. We have data on the importance of this for promoting health from our continuing work with Palette interventions offered to health care staff [24].

The positive ripple effect of the cultural activities was a key finding of this study: "The staffs also need cultural activities", "you can't feel healthier than your caregiver," "Who are we to take care of patients if we can't take care of ourselves"' The idea that the health of the patients is mirrored in the health of the caregiver, signals a need to invest in stress-coping strategies for the health care staff too. Sick leave due to burnout has increased dramatically in recent years and affects caregivers as well [3]. In the future, we propose a need to focus on how to build sustainable health care systems with regard to preventing burnout within both staff and patient groups. According to Missimer et al. [37], a socially sustainable society requires flexibility and adaption/ resilience to survive, something that arts in health and cultural activity interventions may usefully facilitate for health care staff and thus within the system of health care management. The challenge remains in transferring individual knowhow and learning from such interventions to the overall management of the organisation [37]. Trust, among other things, is necessary to this transfer of knowledge $[38,39]$. "We trust each other even though we don't know each other, don't need to know where we come from, trust."

"The health care management have become aware of how culture can be used as a learning process and for future training of caregivers" and "I became curious about connecting cultural activities to caregiving" These quotes signal questions about the concept of the new use of culture in nurse staff training and the health care management started to reflect on synergetic effects by involving culture as part of the staff training. Bringing cultural activities into the health care system triggered creative ideas among the staff and the management. The importance of emotions in learning processes is key [21] and arts and cultural activities may therefore be recommended as a part of healthy learning processes, not only in school contexts.

When we look at the complete interpretation, what emerges overall is the idea that cultural activity provides a potential method of operationalising health sustainability in both the body and more widely in the health care system. This supports Karl Henrik Robèrt's research [40], which argues for a strategic planning for a sustainable health care system. Openness to change and self-awareness of one's mental and physical health are other experiences from the Palette activities that also play an important part in developing a sustainable health care system [41]. Moreover, and as seen in Nielsen et al. [42], employees' appraisal of an intervention itself can play an important role in successful implementation at organisational level.

The results of this study underscore the importance of regarding the health care as a system in which health care staff and patients have an effect on one another. The improved health of the caregiver ripples out to provide a necessary condition for healing in the patient, in other words, to some extent patient health is mirrored in the caregiver. The mirror neuron theory $[42,43]$ describes this idea with reference to areas in the brain, connected to motor areas, that are activated by just watching an activity, as if you were doing it yourself. Rizzolatti et al. [43] suggests that motor actions activate specific parts of the brain and that a more ancient communication system based on recognition of hand and facial gestures, preceding verbal communication.

This study has yielded important insights into the positive effects of cultural intervention in health care not only for patients but for health care staff. We hope that it will inspire further research.

\section{Limitations}

In order to properly situation the contribution of this paper, we discuss its limitations: First of all, there were only four health care centres involved in this study, and they cannot be considered representative of the entire Swedish health care system, although the centres are representative with regard to population, socioeconomic status and size.

Secondly, the participants who shared their experiences all reported positive attitudes towards the Palette. We do not know if the recruitment procedure affected this outcome. The inclusion criteria were related to a cut off over two on the KEDS (Karolinska Exhaustion Disorder Scale) which means that all the patients were burn-out, although the interviewed participants were randomly assigned to the 'culture group', they may have had a pre-existing interest in culture and health which may of course have contributed to the unanimity of positive experiences reported.

Thirdly, the Palette, an intervention of six different cultural activities, represents a wide range of possible experiences. One limit with this study is that we can only consider the experiences from this whole package of cultural activities and cannot evaluate the impact of individual offerings.

Finally, we recognise that it is difficult to secure funding for cultural activities in health care settings. In this Palette study, the centres/ management received the cultural packages for free and this may also have affected the results from the staff in a more positive way.

\section{Conclusion}

Burnout affects many people, especially in the health care sector. We see from the qualitative data produced in this study that the cultural activities can create a more trusting and empathic health care environment where the health of the staff ripples out positively to the patients. This finding speaks to how the arts can have beneficial effects in health care on staff and patients simultaneously and suggests that greater incorporation of the arts into health care may contribute to the development of a more sustainable health care system. 


\section{Ethical Considerations}

All participants consented in writing to participate in the study. The Palette's ethical approval no. is 2012/359 (Uppsala University).

\section{Conflict of Interest}

None of the authors has any conflict of interest toward any aspect of the work described in this manuscript.

\section{References}

1. Grossi G, Perski A, Osika W, Savic I (2015) Stress-related exhaustion disorder - Clinical manifestation of burnout? A review of assessment methods, sleep impairments, cognitive disturbances and neuro-biological and physiological changes in clinical burnout. Scand J Psychol 56: 626-636.

2. Moss M, Good VS, Gozal D, Kleinpell R, SesslerCN (2016) A critical care societies collaborative statement: Burnout syndrome in critical care health-care professionals. A call for action. Am J Respir Crit Care Med 194: 106-113.

3. Swedish Social Insurance Agency (2016) Sjukfrånvarons utveckling 2016 /Sick leave development 2016. Stockholm, Sweden.

4. Rudman A, Gustavsson JP (2011) Early-career burnout among new graduate nurses: A prospective observational study of intra-individual change trajectories. Int J Nurs Stud 48: 292-306.

5. Rudman A, Gustavsson P, Hultell D (2014) A prospective study of nurses' intentions to leave the profession during their first five years of practice in Sweden. Int J Nurs Stud 51: 612-624

6. Golkar A, Johansson E, Kasahara M, Osika W, Perski A, et al. (2014) The influence of work-related chronic stress on the regulation of emotion and on functional connectivity in the brain. PloS ONE 9: e104550.

7. Norlund S, Reuterwall C, Höög J, Lindahl B, Janlert U, et al. (2010) Burnout, working conditions and gender - Results from the northern Sweden MONICA Study. BMC Public Health 10: 1-9.

8. Norlund S, Reuterwall C, Höög J, Janlert, Järvholm L (2015) Work situation and self-perceived economic situation as predictors of change in burnout--a prospective general population-based cohort study. BMC Public Health 15: 329

9. Zoni S, Lucchini RG (2012). European approaches to work-related stress: A critical review on risk evaluation. Saf Health Work 3: 43-49.

10. Åsberg M, Krakau I, Nygren Å, Rodhe M, Wahlberg A, et al. (2010) Stress som orsak till psykisk ohälsa/stress as the cause of mental illness. Läkartidningen 107: 1307-1310.

11. Hayes B, Bonner A, Pryor J (2010) Factors contributing to nurse job satisfaction in the acute hospital setting: A review of recent literature. J Nurs Manag 18: 804-814.

12. Kutney-Lee A, Wu ES, Sloane DM, Aiken LH (2013) Changes in hospital nurse work environments and nurse job outcomes: An analysis of panel data. Int Nurs Stud 50: 195-201.

13. Shanafelt TD, Hasan O, Dyrbye LN, Sinsky C, Satele D, et al. (2015) Changes in burnout and satisfaction with work-life balance in physicians and the general US working population between 2011 and 2014. Mayo Clin Proc 90: 1600-1613.

14. Grape Viding C, Osika W, Theorell T, Kowalski J, Hallqvist J, et al. (2015) "The Culture palette"- A randomized intervention study for women with burnout symptoms in Sweden. Br J Med Pract 8: 813-821.

15. Theorell T, Osika W, Leineweber C, Magnusson Hanson LL, Bojner Horwitz E, et al. (2013) Is cultural activity at work related to mental health in employees? Int Arch Occup Environ Health 86: 281-288.

16. Clift SM, Hancox G (2001) The perceived benefits of singing: Findings from preliminary surveys of a University College Choral Society. J Royal Soc Prom Health 121: 248-256.

17. Cohen GD, Perlstein S, Chapline J, Kelly J, Firth KM, et al. (2006) The impact of professionally conducted cultural programs on the physical health, mental health and social functioning of older adults. Gerontologist 467: 26-34.

18. Theorell T, Konarski K, Engström R, Lagercrantz AM, Teszåry J, et al. (1993) Treatment of long-term psychosomatic states with creative art psychotherapy. Vard 1: 94-97.

19. Theorell T (2014) Psychological health effects of musical experiences Theories, studies and reflections in music health science. Springer.

20. Bojner Horwitz E, Lennartsson AK, Theorell TPG, Ullén F (2015) Engagement in dance is associated with emotional competence in interplay with others. Front Psychol 6: 1096.

21. Immordino-Yang MH, McColl A, Damasio H, Damasio A (2009) Neural correlates of admiration and compassion. Proc Natl Acad Sci U S A 106: 8021-8026.

22. Bygren LO, Weissglas G, Wikström BM, Konlaan BB, Grjibovski A, et al. (2009) Cultural participation and health: A randomized controlled trial among medical care staff. Psychosom Med 71: 469-473.

23. Repar P, Patton D (2007) Stress reduction for nurses through arts-inmedicine at the university of New Mexico hospitals. Holistic Nurs Pract 182-184.

24. Bojner Horwitz E, Huss E (2017) Using internet based arts to promote inter-generational meetings between young people and senior citizens. J Appl Arts Health 7: 297-311.

25. Thomson LJ, Ander, EE, Menon U, Lanceley A, Chatterjee HJ (2012) Enhancing cancer patient well-being with a non-pharmacological, heritage-focused intervention. J Pain Symp Man 44: 731-740.

26. Bojner Horwitz E, Theorell T, Anderberg UM (2003) Fibromyalgia patients' own experiences of video self-interpretation: A phenomenological-hermeneutic study. Scand J Car Sci 17: 257-264.

27. Lindseth A, Norberg A (2004) A phenomenological hermeneutical method for researching lived experience. Scand J Car Sci 18: 145-153.

28. Csikszentmihalyi M (2000) Happiness, flow and economic equality. Am Psychol 55: 1163-1164.

29. Craig P, Dieppe P, Macintyre S, Michie S, Nazareth I, et al. (2008) Developing and evaluating complex interventions: The new Medical Research Council guidance. BMJ Clin Res Ed 337: a1655.

30. Craig P, Petticrew M (2013) Developing and evaluating complex interventions: Reflections on the 2008 MRC guidance. Int J Nurs Stud 50: 585-587.

31. Davies CR, Knuiman M, Wright P, Rosenberg M (2014) The art of being healthy: a qualitative study to develop a thematic framework for understanding the relationship between health and the arts. BMJ Open 4: e004790.

32. Fancourt D, Aufegger L, Williamon A (2015) Low-stress and high-stress singing have contrasting effects on glucocorticoid response. Front Psychol 6: 1242 .

33. Stenfors CUD, Marklund P, Magnusson Hanson LL, Theorell T, Nilsson LG (2013) Subjective cognitive complaints and the role of executive cognitive functioning in the working population: a case-control study. PloS ONE 8: e83351.

34. LeDoux JE (1998) The emotional brain: The mysterious underpinnings of emotional life. Weidenfeld and Nicolson, New York.

35. Bojner Horwitz E, Stenfors C, Osika W (2013) Contemplative inquiry in movement: Managing writer's block in academic writing. Int J Transpers Stud 32: 16-26.

36. Baumgartner T, Lutz K, Schmidt CF, Jäncke L (2006) The emotional power of music: How music enhances the feeling of affective pictures. Brain Res 1075: 151-164.

37. Missimer M, Robèrt KH, Broman G, Sverdrup H (2010) Exploring the possibility of a systematic and generic approach to social sustainability. J Clean Prod 18: 1107-1112.

38. Augustsson H, Törnquist A, Hasson H (2013) Challenges in transferring individual learning to organizational learning in the residential care of older people. J Health Org Man 27: 390-408.

39. Missimer M, Robèrt KH, Broman G (2016) A strategic approach to social sustainability - part 1: Exploring the social system. J Clean Prod. 
Citation: Viding CG, Osika W, Horwitz EB (2017) "You Can't Feel Healthier than Your Caregiver" - The Ripple Effect of Trust and Empathy for Patients and Health Care Staff, Cultivated through Cultural Activities. J Nurs Care 6: 422. doi:10.4172/2167-1168.1000422

Page 8 of 8

40. Missimer M, Robèrt KH, Broman, G (2016) A strategic approach to social sustainability - Part 2: A principle-based definition. J Clean Prod.

41. Robèrt KH, Daly H, Hawken P, Holmberg J (1997) A compass for sustainable development. Int J Sust Develop and World Ecol 4: 79-92.
42. Nielsen K, Randall R, Albertsen K (2007) Participants' appraisals of process issues and the effects of stress management interventions. J Org Behav 28: 793-810.

43. Rizzolatti G, Fadiga L, Gallese V, Fogassi L (1996) Premotor cortex and the recognition of motor actions. Cogn Brain Res 3: 131-141. 